$$
\text { "kosztolanyi" — 2005/2/17 — 17:02 — page 275 — \#1 }
$$

\title{
Decomposition of triangles into isosceles triangles II Complete solution of the problem by using a computer
}

\author{
József Kosztolányi, Zoltán KovÁcs and ERzsébet Nagy
}

Abstract. We solve an open decomposition problem in elementary geometry using pure mathematics and a computer programme, utilizing a computer algebra system.

Key words and phrases: triangle, isosceles triangle, obtuse triangle, decomposition, computer algebra, batch processing.

ZDM Subject Classification: G43, H33, K23, R20.

\section{Introduction}

In our article Decomposition of Triangles Into Isosceles Triangles I [1] we wrote about how we explored the problem of what triangles can be decomposed into a certain number of isosceles triangles with some interested and able pupils of 13 and 14 in a maths camp. We determined all triangles that can be decomposed into two isosceles triangles and we proved that any triangle can be decomposed into $n$ isosceles triangles if $n \geq 4$. The question of what triangles can be decomposed into three isosceles triangles also arose. We could only partially solve this problem in the camp, namely, we proved that any non-obtuse triangle and also obtuse isosceles triangles can be decomposed into three isosceles triangles.

In the following article it will be shown that every obtuse scalene triangle can be decomposed into three isosceles triangles. Because of the large number of

Copyright (C) 2004 by University of Debrecen

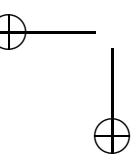


essentially different decompositions we used a computer to explore certain ways of decomposition.

Using the usual notation for the angles of triangle $A B C$ we assume that $\alpha>90^{\circ}>\beta>\gamma$ and we express the angles of the triangles with $\gamma$.

\section{Case A: The obtuse angle is not decomposed}

First those obtuse scalene triangles are given which can be decomposed into three isosceles triangles so that the cutting line does not contain the vertex at the obtuse angle. It is easy to see that in this case there are two possibilities:

1. A cutting line containing vertex $B$ cuts the isosceles triangle $A B D$ from the original triangle (Figure 1).

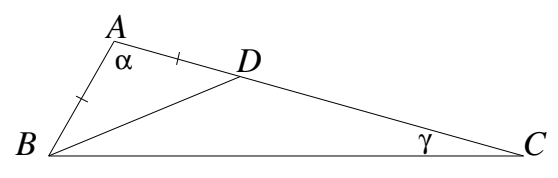

Figure 1.

2. A line containing some inner point $D$ of side $A B$ cuts the isosceles triangle $A D E$ from the original triangle (Figure 2).

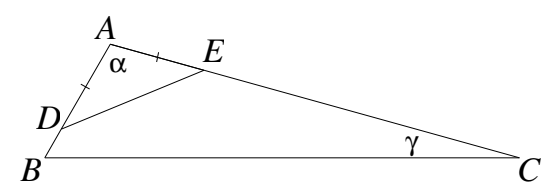

Figure 2.

\subsection{The analysis of Case 1}

We have to examine when triangle (Figure 1) $B C D$ can be decompose into two isosceles triangles with the assumptions for the angles of the original triangle. There are several different ways of decomposing the triangle depending upon which vertex is on the cutting line and which line segments will be of equal length after the decomposition. 


\subsubsection{1/a)}

The cutting line passes through vertex $B$ and intersects side $C D$ in point $E$. Now since angle $E D B$ and angle $C E B$ are obtuse, only $B D=D E$ and $B E=E C$ can hold (Figure 3).

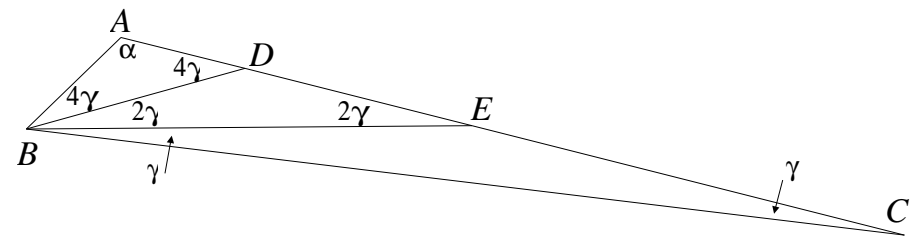

Figure 3.

Figure 3 shows that $E B C \angle=B C E \angle=\gamma, D B E \angle=B E D \angle=2 \gamma$ and $A B D \angle=B D A \angle=4 \gamma$. It follows that $\alpha=180^{\circ}-8 \gamma$ and $\beta=7 \gamma$. It has been assumed that $\alpha>90^{\circ}$, therefore

$$
180^{\circ}-8 \gamma>90^{\circ}
$$

and thus

$$
\gamma<11,25^{\circ}
$$

So if $\gamma<11,25^{\circ}$, then the triangle with angles $180^{\circ}-8 \gamma, 7 \gamma, \gamma$ can be decomposed into three isosceles triangles as seen on Figure 3.

\subsection{2. $1 / \mathrm{b})$}

The cutting line passes through vertex $C$ and intersects side $B D$ in point $E$. Since angles $C D E$ and $C E B$ are obtuse, it follows that $B E=E C$ and $E D=D C$ (Figure 4).

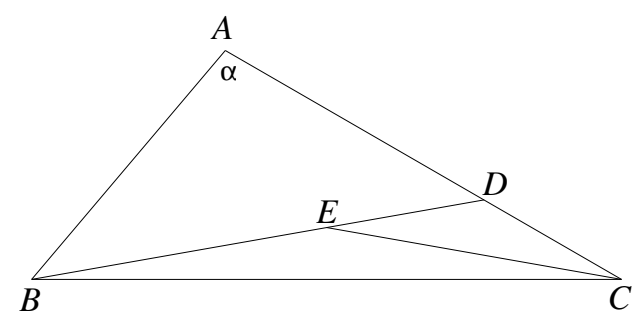

Figure 4. 
¿From Figure $4 B D A \angle=90^{\circ}-\frac{\alpha}{2}$ and therefore $E C D \angle=D E C \angle=45^{\circ}-\frac{\alpha}{4}$. Since $C E B \angle+D E C \angle=180^{\circ}, C E B \angle=135^{\circ}+\frac{\alpha}{4}$. It follows that $B C E \angle=$ $22,5^{\circ}-\frac{\alpha}{8}$. Thus

$$
\gamma=45^{\circ}-\frac{\alpha}{4}+22,5^{\circ}-\frac{\alpha}{8}=67,5^{\circ}-\frac{3 \alpha}{8}
$$

which gives $\alpha=180^{\circ}-\frac{8}{3} \gamma$ and $\beta=\frac{5}{3} \gamma \cdot \alpha$ is obtuse, that is $180^{\circ}-\frac{8}{3} \gamma>90^{\circ}$, so $\gamma<33,75^{\circ}$.

It follows that if $\gamma<33,75^{\circ}$, then the triangle with angles $180^{\circ}-\frac{8}{3} \gamma$, $\frac{5}{3} \gamma, \gamma$ can be decomposed into 3 isosceles triangles as shown in Figure 4 .

\subsection{3. $1 / \mathrm{c}$ )}

The cutting line passes through vertex $D$ of triangle $B C D$ and intersects side $B C$ in point $E$ so that angle $D E C$ is obtuse. Then $D E=E C$ (Figure 5).

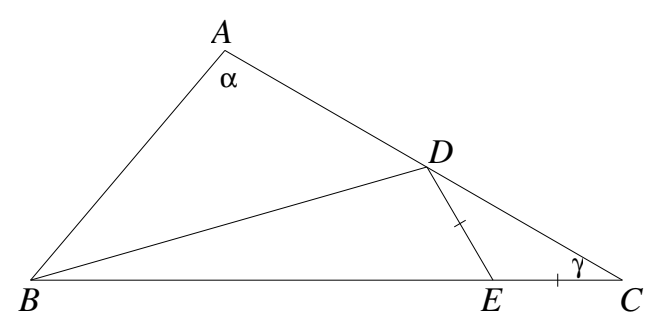

Figure 5.

Triangle $B E D$ has to be isosceles. There are three possibilities for this:

- $B E=E D$. From Thales' theorem, angle $C D B$ has to be a right angle, which contradicts the fact that angle $C D B$ is obtuse. Thus such a decomposition is impossible.

- $D B=B E$. Figure 6 shows that then $B D A \angle+3 \gamma=180^{\circ}$. As $B D A \angle<45^{\circ}$, $\gamma>45^{\circ}$. This, however, contradicts the assumptions made on the angles of triangle $A B C$, therefore such a decomposition is also impossible.

- $B D=D E$. Then Figure 7 shows that $D B E \angle=B E D \angle=2 \gamma$. Angle $B D A$ is an outer angle of triangle $B C D$, thus $B D A \angle=A B D \angle=3 \gamma$. It follows that $\alpha=180^{\circ}-6 \gamma, \beta=5 \gamma$, and since $\alpha>90^{\circ}, 180^{\circ}-6 \gamma>90^{\circ}$, that is $\gamma<15^{\circ}$.

We get that if $\gamma<15^{\circ}$, then a triangle with angles $180^{\circ}-6 \gamma, 5 \gamma, \gamma$ can be decomposed into 3 isosceles triangles as shown in Figure 7. 


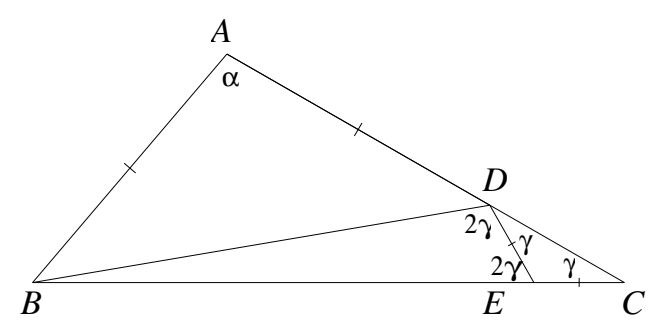

Figure 6 .

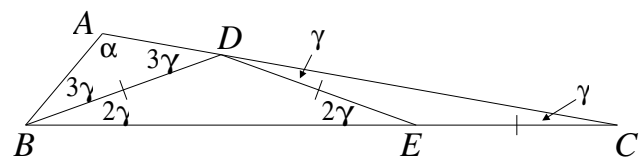

Figure 7.

\subsection{4. $1 / \mathrm{d})$}

The cutting line passes through vertex $D$ of triangle $B C D$ and intersects side $B C$ in point $E$ for which angle $B E D$ obtuse. Then $B E=E D$ (Figure 8).

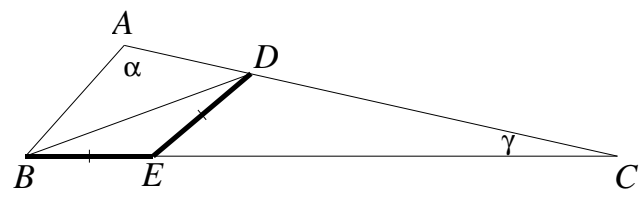

Figure 8.

Again, there are three possibilities for triangle $C D E$ to be isosceles.

- $D E=E C$. This is not possible as angle $C D B \angle$ would be a right angle.

- $E C=C D$. Then from Figure 9 expressing the angles with $\gamma$ it follows that $C E D \angle=C D E \angle=90^{\circ}-\frac{\gamma}{2}, B E D \angle=90^{\circ}+\frac{\gamma}{2}, E B D \angle=45^{\circ}-\frac{\gamma}{4}$, that is $B D A \angle=45^{\circ}-\frac{\gamma}{4}+\gamma=45^{\circ}+\frac{3 \gamma}{4}>45^{\circ}$. As $\alpha>90^{\circ}, B D A \angle>45^{\circ}$ is impossible. Therefore such a decomposition does not exist, either. 


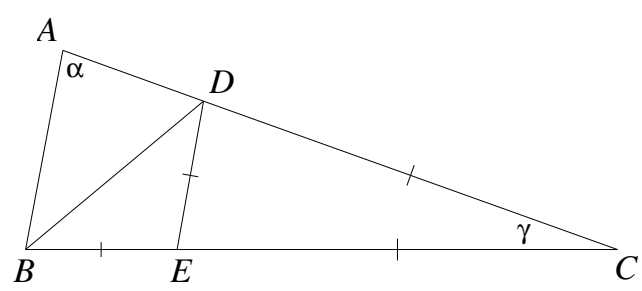

Figure 9.

- $E D=D C$. Then from Figure $10 D E C \angle=\gamma$, so $D B E \angle=E D B \angle=\frac{\gamma}{2}$. Angle $B D A$ is an outer angle of triangle $B C D$, so $B D A \angle=\frac{3 \gamma}{2}$ and $\alpha \stackrel{2}{=}$ $180^{\circ}-3 \gamma \cdot \alpha>90^{\circ}$ holds if and only if $\gamma<30^{\circ}$.

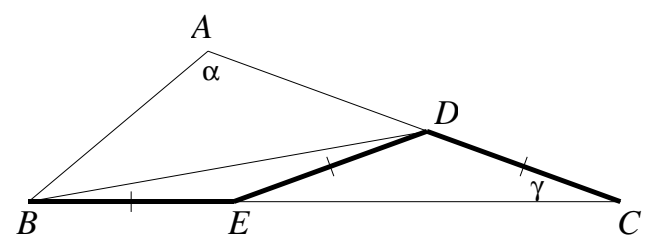

Figure 10.

Thus if $\gamma<30^{\circ}$, a triangle with angles $180^{\circ}-3 \gamma, 2 \gamma, \gamma$ can be decomposed into isosceles triangles as shown in Figure 10.

Note: Since angle $C D B$ is obtuse, cutting it with a line which is perpendicular to $B C$ and passed through $D$ we get two right triangles which cannot be isosceles at the same time.

It has been shown that scalene obtuse triangles with the following angles can be decomposed into three isosceles triangles (the angles are listed in increasing order):

(a) $180^{\circ}-8 \gamma, 7 \gamma, \gamma ; \quad \gamma<11,25^{\circ}$

(b) $180^{\circ}-\frac{8}{3} \gamma, \frac{5}{3} \gamma, \gamma ; \quad \gamma<33,75^{\circ}$

(c) $180^{\circ}-6 \gamma, 5 \gamma, \gamma ; \quad \gamma<15^{\circ}$

(d) $180^{\circ}-3 \gamma, 2 \gamma, \gamma ; \quad \gamma<30^{\circ}$

\subsection{Analysis of Case 2}

From the original triangle $A B C$ now isosceles triangle $A D E$ is chopped off (Figure 2), so we need to determine quadrangles $B C E D$ which can be decomposed 
into two isosceles triangles and which also satisfy the assumptions made about the angles. A quadrangle can be decompose into two triangles if and only if we cut it by one of its diagonals. Thus there are two possibilities depending upon which diagonal is used for this purpose.

\subsection{1. $2 / a)$}

We cut by diagonal $B E$. Then, since angle $E D B$ is obtuse, $B D=D E$ (Figure 11).

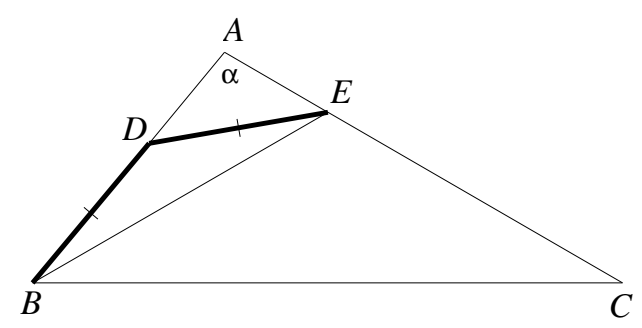

Figure 11 .

There are essentially three different possibilities for triangle $B C E$ to be isosceles.

- $B E=E C$. Then if $B E D \angle=D B E \angle=\varepsilon$, then $A D E \angle=D E A \angle=2 \varepsilon$ and so $3 \varepsilon=2 \gamma$. From this $\varepsilon=\frac{2}{3} \gamma$, therefore $\beta=\varepsilon+\gamma=\frac{5}{3} \gamma$. Thus, the angles of triangle $A B C$ are $180^{\circ}-\frac{8}{3} \gamma, \frac{5}{3} \gamma, \gamma$. This is not a new result: it has already been found during the analysis of Case 1/b (Figure 4), where we also determined that our assumptions about the angles hold if $\gamma<33,75^{\circ}$. It can be concluded that triangles with angles $180^{\circ}-\frac{8}{3} \gamma, \frac{5}{3} \gamma, \gamma\left(\gamma<33,75^{\circ}\right)$ can be decomposed into 3 isosceles triangles at least two different ways.

- $B C=C E$. Figure 12 shows that in this case $E B C \angle=C E B \angle=90^{\circ}-\frac{\gamma}{2}$. However, this is not possible since $\alpha>90^{\circ}$ and therefore $\alpha+A B E \angle=$ $90^{\circ}-\frac{\gamma}{2}>90^{\circ}$ should hold.

- $E B=B C$. Then $\gamma>\alpha$ would be true which is impossible with our assumptions.

\subsection{2. $2 / \mathrm{b})$}

The quadrangle is cut by diagonal $C D$. Then angle $C E D$ is obtuse, so $D E=E C$ (Figure 13). 


$$
\text { "kosztolanyi" — 2005/2/17 — 17:02 — page } 282 \text { - \#8 }
$$

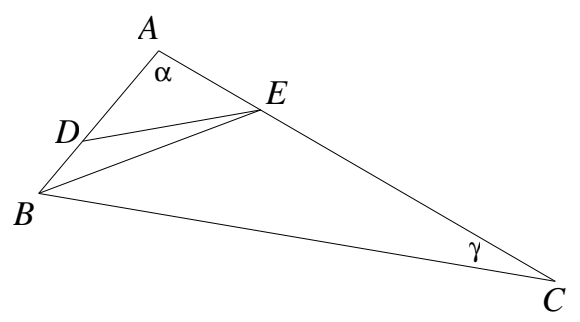

Figure 12.

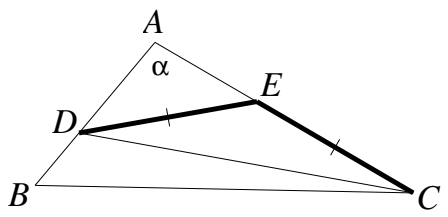

Figure 13.

Figure 13 shows that $C D B \angle=\alpha+D C A \angle>\alpha>90^{\circ}$, which leaves $B D=$ $D C$ as the only possibility. In this case, however, $\gamma>\beta$ would hold, which contradicts the assumption made about the angles, therefore such a decomposition is impossible for every triangle.

To sum up, we can conclude that no new triangles have been found during the analysis of Case 2. This means that those scalene triangles that can be decomposed into 3 isosceles triangles without dividing their obtuse angle are of type (a), (b), (c) or (d).

3. Case B: The obtuse angle is divided - utilizing a computer

In the previous section it was assumed that the obtuse angle is not divided. Now we examine the remaining cases. Because of the large number of cases to be analyzed we use a personal computer.

\subsection{Apparatus}

Three techniques are mixed.

- The solution involves more than 100 systems of linear equations and a lot more linear equations. This is done by the computer algebra system Maxima 
which has been developed at MIT since 1965 as Macsyma but made public by William Schelter in 1997.

- The inner logic of the equation solver programme is written in Pascal. This is probably the best programming language to describe mathematical thinking. In the actual implementation Free Pascal is used.

- The communication between the Pascal programme and the Maxima system is maintained with simple Unix commands. For this purpose the free operation system Linux is used.

Each of the three programmes are freeware, that is they are accessible for everyone free of charge. The problem could be solved using other programming languages and computer algebra systems (maybe even in a simpler way), but one of our main points was to make sure that the whole range of our devices can be easily accessed by the Reader.

\subsection{Algebraic formalization}

First, we introduce some new notation. The computer programme uses Latin letters and no Greek letters, so we will denote the angles with the letters of the English alphabet: $a, b, \ldots$ Depending whether the line dividing the obtuse angle reaches side $B C$ there are two parts of the problem.

3.2.1. The obtuse angle is divided with a line that reaches side $B C$ ("long cut")

Unlike before, we denote the angle at vertex $B$ with $a$ and the angle at vertex $A$ with $b$. The sum of the inner angles of a triangle is apparently

$$
a+b+c=180^{\circ}
$$

and since the angle at vertex $A$ is obtuse,

$$
90^{\circ}<b<180^{\circ}
$$

and therefore

$$
0^{\circ}<a<90^{\circ}
$$

and

$$
0^{\circ}<c<90^{\circ}
$$

also holds. 
In the following three steps the remaining equations of our system of equations are determined:

1. Triangle $A B C$ is decomposed into two triangles with a line passing through vertex $A$. Thus we divide angle $b$ into angles $d$ and $e$ :

$$
b=d+e
$$

There are two additional angles: let $D$ denote the intersection of the cutting line and side $B C$ and let $f$ and $g$ denote angles $B D A \angle$ and $C D A \angle$ respectively. Obviously

$$
\begin{aligned}
a+d+f & =180^{\circ}, \\
c+e+g & =180^{\circ}, \\
f+g & =180^{\circ} .
\end{aligned}
$$

2. We do not violate the generality if we assume that the other dividing line segment is inside the triangle $A C D$. This line segment will obviously divide angle $c, e$ or $g$ further. Thus, in an algebraic formulation, one of the three following equalities must hold:

$$
c=h+i
$$

or

$$
e=h+i
$$

or

$$
g=h+i
$$

On the opposing side we get a new point and two new angles. Let them be denoted by $E, j$ and $k$, respectively. It is obvious that

$$
j+k=180^{\circ},
$$

and, depending upon which above angle is given by $h+i$ one of the following pairs of equalities must also hold:

$$
\begin{aligned}
& h+e+j=180^{\circ}, \\
& i+g+k=180^{\circ},
\end{aligned}
$$

or

$$
\begin{aligned}
h+g+j & =180^{\circ}, \\
i+c+k & =180^{\circ},
\end{aligned}
$$




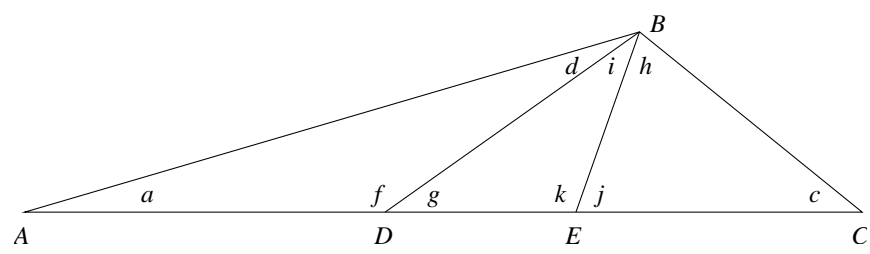

Figure 14 .

or

$$
\begin{aligned}
& h+c+j=180^{\circ} \\
& i+e+k=180^{\circ}
\end{aligned}
$$

(In Figure 14, one of the 81 possible cases is shown: Angle $b$ equals $B A C \angle$, while angle $e$ equals $D A C \angle$. This leads to the following system of equations: $a+b+c=180^{\circ}, b=d+e, f+g=180^{\circ}, a+d+f=180^{\circ}$, $c+e+g=180^{\circ}, e=h+i, j+k=180^{\circ}, h+c+j=180^{\circ}, i+g+k=180^{\circ}$, $d=a, h=j, g=i$. The solution of the system is $b=7 a, c=180^{\circ}-8 a$, $d=a, e=6 a, f=180^{\circ}-2 a, g=2 a, h=4 a, i=2 a, j=4 a, k=180^{\circ}-4 a$. Also, from the system of inequalities $\frac{90^{\circ}}{7}<a<\frac{45^{\circ}}{2}$ must hold for $a$.) We note that each of the resulting angles must be greater than $0^{\circ}$, but smaller than $180^{\circ}$. Thus

$$
0^{\circ}<x<180^{\circ}
$$

where $x \in\{d, e, f, g, h, i, j, k\}$.

3. The three resulting triangles (which make up triangle $A B C$ ) have to be isosceles. This is ensured by making a pair of angles equal for each triangle. For example, for triangle $A B D$

$$
a=d
$$

or

$$
a=f
$$

or

$$
d=f .
$$

We do the same with the other two triangles. 


\subsection{2. "Short cut"}

A slightly different notation is used here for the angles of the triangle. It is introduced to help the computer analysis.

First, triangle $A B C$ is decomposed into triangles $A B D$ and $B C D$ with a line segment passing through $B$; of course, this line segment could pass through $C$ instead of $B$, but generality is not violated here (Figure 15). Then triangle $A B D$ is decomposed with a line segment passing through $A$. Thus $b$ is once again divided into angles $d$ and $e$ :

$$
b=d+e .
$$

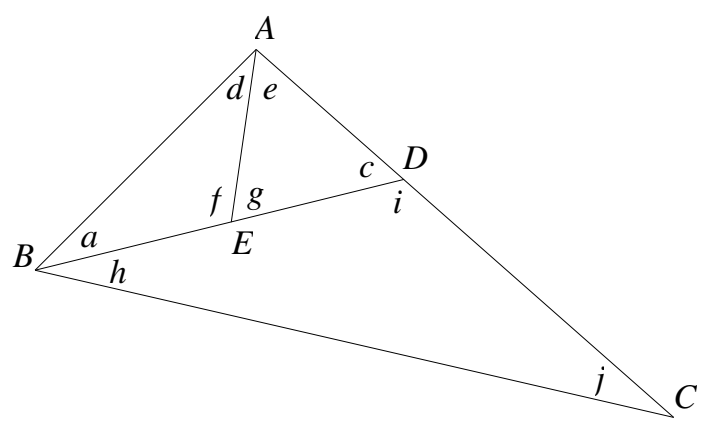

Figure 15.

Let $a$ and $c$ denote angles $D B A \angle$ and $B D A \angle$, respectively. Thus we recreate the "long cut" case in such a way that triangle $B D C$ is attached to the side $B C$ of that case.

The sum of the inner angles of triangle $A B D$ obviously

$$
a+b+c=180^{\circ}
$$

and since the angle at vertex $A$ is obtuse,

$$
90^{\circ}<b<180^{\circ} \text {. }
$$

Similarly to the "long cut" case, new angles are formed here: let $E$ denote the intersection of the line segment dividing $A$ and side $B D$; let angles $B E A \angle$ and $D E A \angle$ be denoted by $f$ and $g$. Again, obviously,

$$
\begin{aligned}
a+d+f & =180^{\circ}, \\
c+e+g & =180^{\circ}, \\
f+g & =180^{\circ} .
\end{aligned}
$$


In the following 3 steps we determine the system of equations that provides the solution:

1. Let $h, i$ and $j$ the angles of triangle $B D C$ ("attached to" side $B D$ ). Let $k=C B A \angle$. Clearly,

$$
\begin{aligned}
h+i+j & =180^{\circ}, \\
c+i & =180^{\circ}, \\
k & =a+h .
\end{aligned}
$$

2. Again, it is clear that all angles must be greater than $0^{\circ}$ and smaller than $180^{\circ}$. Thus,

$$
0^{\circ}<x<180^{\circ}
$$

where $x \in\{a, c, d, e, f, g, h, i\}$. Observe here that the roles of $a$ and $c$ as well as $k$ and $j$ are reversed if compared to the "long cut" case.

3. The three resulting triangles (which make up triangle $A B C$ ) are all supposed to be isosceles. This is proved just like in the "long cut" case.

3.3. Rewriting the inequalities as equations and solving the system of equations and inequalities

Unfortunately, Maxima does not support the simple solution of systems of inequalities, so we also need to represent them with equations.

During this, first the equations are given, then every single angle is expressed in terms of $a$. It is, for example, quite trivial that in the "long cut" case the for each angle $x(x \in\{b, c, d, e, f, g, h, i, j, k\})$ a linear equation of the form

$$
x=A \cdot a+B
$$

is derived where $A$ and $B$ are given real numbers. Since $0^{\circ}<x<180^{\circ}$ (moreover, if $x=b$, then $90^{\circ}<b<180^{\circ}$ and if $x=c$, then $0^{\circ}<c<90^{\circ}$ also holds), $x=0^{\circ}$ and $x=180^{\circ}$ using the method of substitution, after rearranging the equation we get two such extrema for $a$ which it cannot assume: in case $x=0^{\circ} a=-B / A$ and in case $x=180^{\circ} a=\left(180^{\circ}-B\right) / A$.

¿From these latter results $a$ can be confined in between two boundaries it cannot assume, that $a \in\left(\min \left(-B / A,\left(180^{\circ}-B\right) / A\right), \max \left(-B / A,\left(180^{\circ}-B\right) / A\right)\right)$ type requirements can be given. If the section of the intervals in all these assumptions is not empty, then the actual system of equations and inequalities can be solved; it can be found for each $x$ after determining a possible solution $a$. 
In actual practice, Maxima determines this interval, so the programme code does not contain the determination of $A$ and $B$.

\subsection{The number of solutions}

In the "long cut" case point $E$ can be chosen in three different ways depending upon whether angle $c, e$ or $g$ is divided. Also, the three resulting triangles can all be isosceles in three different ways. Thus the following system of equations and inequalities is to be examined: $3 \cdot 3^{3}=3^{4}=81$. Some of these are certainly inconsistent, which shows in the fact that the section of intervals in connection with $a$ will be empty.

In the "short cut" case point $E$ can be chosen in only one way. Therefore it generates only $3^{3}=27$ cases.

When the programme finished running, only 24 out of 81 cases, and 4 out of 27 "short cut" cases proved to be adequate.

\subsection{Only 15 of the 24 solutions are different}

Some cases in the "long cut" case type solutions will be essentially the same. This follows primarily from the fact that when decomposing the obtuse angle into three angles the cases when the roles of $a$ and $c$ are reversed come up twice. On the other hand, the number of decompositions in case of a given triangle is not important: these cases are to be considered identical. Also, if a solution is a special case of a previous solution it cannot count as a separate solution.

The possible types of solutions are shown on Figure 16, Figure 17 and Figure 18; every detail of these figures was created by a computer. The angle at vertex $A$ can be seen "on the top", the angle at vertex $B$ "on the left", vertex $C$ "on the right". The computer programme found some ideas of filtering which our figures are based upon while running. These are the following (in parentheses an "identifying" type number is given. It is used within the computer programme for classifying cases that are essentially the same):

- Case \#17 gives the same set of triangles as case \#10, although the way it is decomposed is different (type 1).

- Case \#13 is the reflection of case \#4, and the way it is decomposed is the same. Similarly, case \#14 is equivalent to cases \#11, \#16 and \#5; while case \#23 to case \#21 (type 2). 
- Case \#22 is the reflection of case \#2, but with a different decomposition (type 2).

- The set of triangles in case \#9 is a subset of the set of triangles in case \#4, that is case \#9 is a special case of case \#4, although the decomposition is different (type 3 ).

- The set of triangles in case \#15 is a subset of the set of triangles in case \#6, that is case \#15 is a special case of case \#6, although the decomposition is different (type 3).

- Reflecting the set of triangles in case \#18, the resulting set of triangles is a subset of the triangles in case \#1, therefore case \#18 is a special case of case \#1, although the decomposition is different (type 4 ).
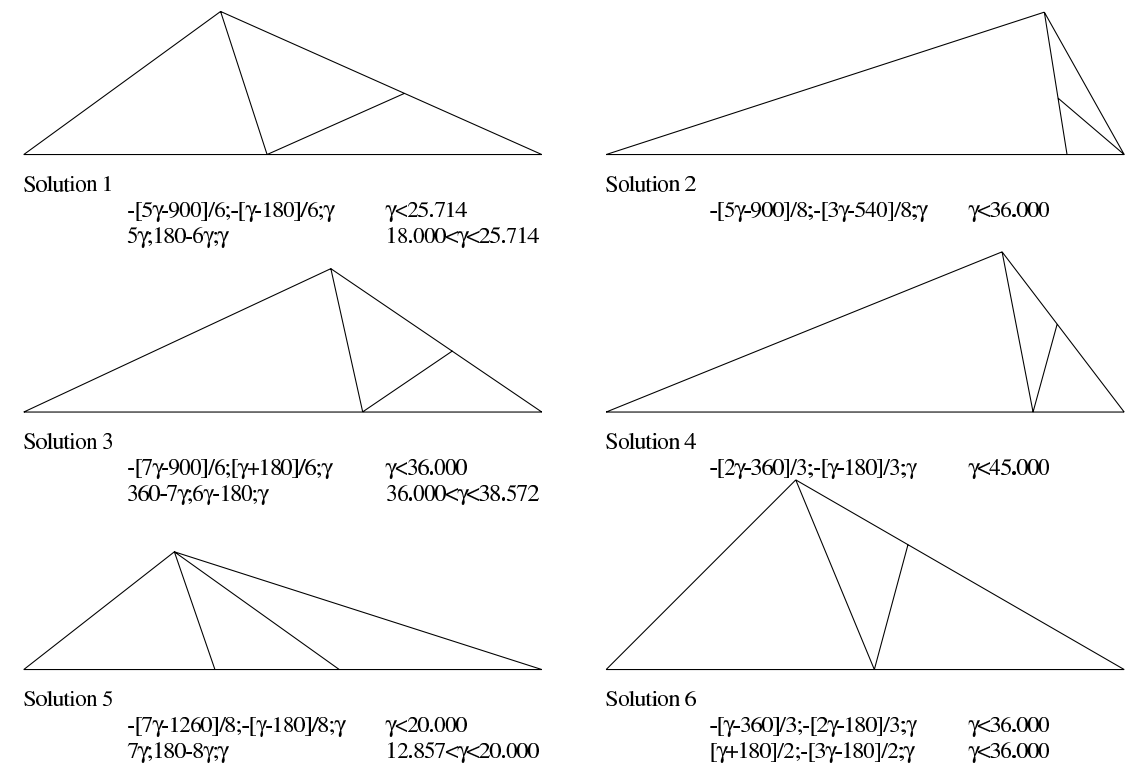

Figure 16. The solutions of the problem (1-6.)

The triangles in case \#19 are isosceles. As the examination of symmetric triangles was not among our original objective in this section, this case is not taken into account (though it is part of the final summary, of course).

The detailed analysis of all 81 cases can be found in the computer programme, which is downloadable from the internet. 
$\bigoplus$

"kosztolanyi" - 2005/2/17 - 17:02 — page $290-\# 16$
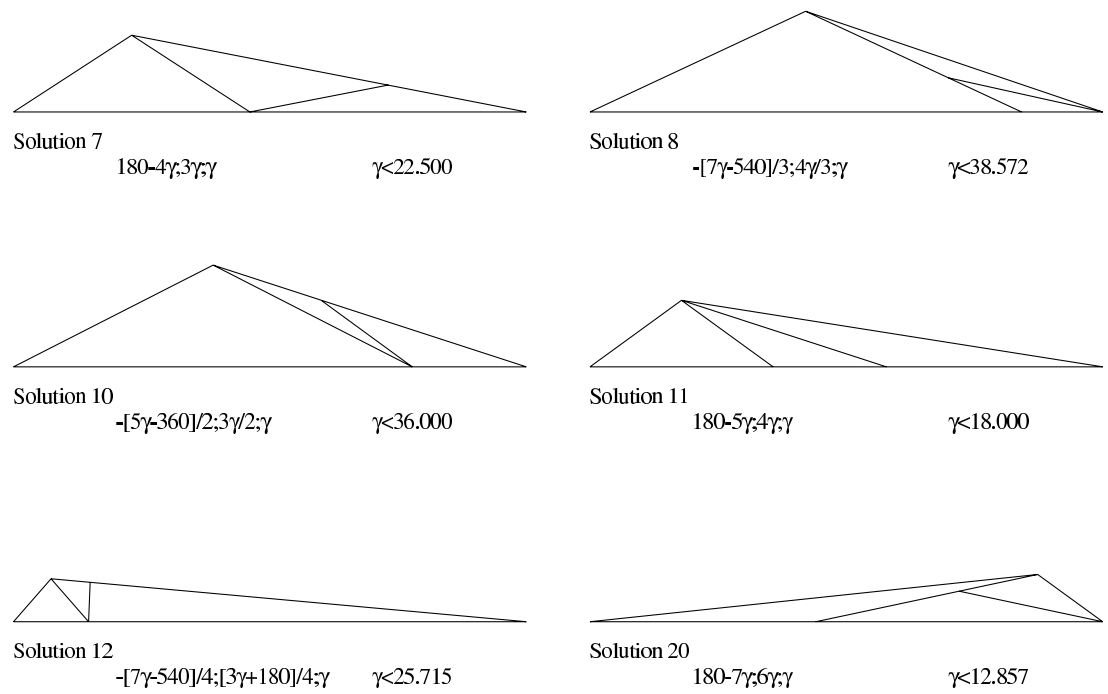

Figure 17. The solutions of the problem (7-20.)
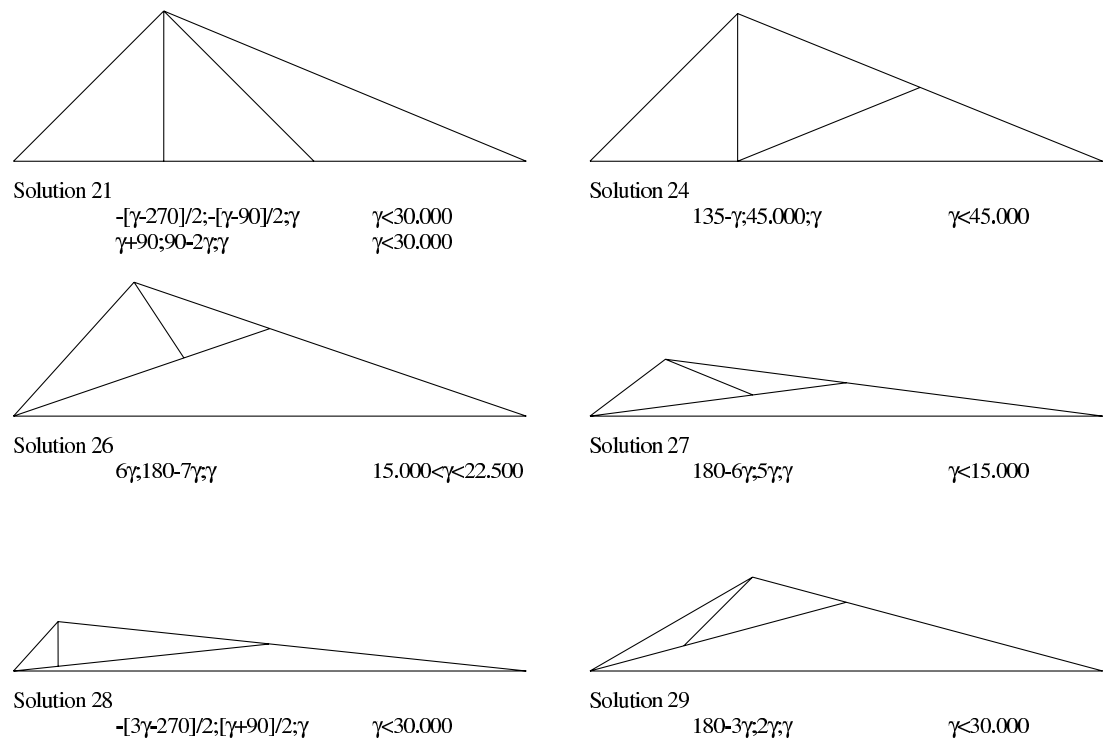

Figure 18. The solutions of the problem (21-29.) 
Besides the resulting 4 "short cut" solutions there is actually a fifth one, which would determine a degenerate triangle (this is case \#25). The actual solutions are numbered from 26 to 29.

\subsection{The answer}

There is a last step to take after sorting out the cases. Here we need to examine the remaining 14 solutions, so that if the smallest angle is denoted by $\gamma$, the second smallest by $\beta$ and the largest (the obtuse one) by $\alpha$, how can $\alpha$ and $\beta$ expressed with $\gamma$. The only problem here comes from the fact that from $a$ and $c$ sometimes only $a$, sometimes only $c$, sometimes both of them can be $\gamma$. In this latter case our solution will have two parts.

¿From a mathematical point of view comparing $a$ and $c$ is not very difficult since their ratio is constant. That is, depicting their relation in an $a-c$ coordinate system, and comparing the resulting line segment with the line $c=a$, if they do not intersect, only $a$ or only $c$ will be $\gamma$ in the given solution, otherwise both of them. (Figure 19 shows two examples for the determination of $\gamma$. In case $c=f(a)$ the solution falls into two partial solutions, while in case $c=g(a)$ as, in the whole set of triangles, $a<c$ from $a$ and $c \gamma=a$ holds.)

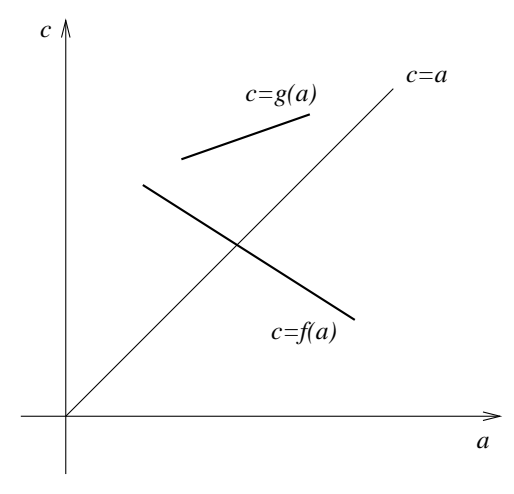

Figure 19.

On Figures 16, 17 and 18 it can be seen that while in cases \#1, 3, 5, 6 and 21 there will be 2 different solutions, in cases \#2, 4, 7, 8, 10, 11, 12, 20 and 24 there will be only one. That is 19 cases altogether. Adding the "short cut" cases to these, there are 23 essentially different types of obtuse triangles that can be 
decomposed into 3 isosceles triangles if we divide the obtuse angle and do not take the trivial case of an "isosceles" triangle into consideration.

The solution types found by the computer are listed in Table 1.

Table 1. The computer programme that gives the full answer uses computer algebraic calculations for the solution of 1307 partial problems. It took 2 minutes and 58 seconds to run on a $600 \mathrm{MHz}, 256 \mathrm{MB}$, Pentium III with SuSE Linux 7.3 and 1 minute 29 seconds on a 2800 $\mathrm{MHz}, 2 \mathrm{~GB}$, Pentium IV with SuSE Linux 9.0.

\begin{tabular}{|c|c|c|c|c|c|}
\hline$\#$ & Solution & $\alpha$ & $\beta$ & $\gamma$ & Condition \\
\hline \hline 1 & $1 / \mathrm{a}$ & $180^{\circ}-\frac{5}{6} \gamma$ & $30^{\circ}-\frac{\gamma}{6}$ & $\gamma$ & $\gamma<\frac{180^{\circ}}{7}$ \\
\hline 2 & $1 / \mathrm{b}$ & $5 \gamma$ & $180^{\circ}-6 \gamma$ & $\gamma$ & $18^{\circ}<\gamma<\frac{180^{\circ}}{7}$ \\
\hline 3 & 2 & $112,5^{\circ}-\frac{5}{8} \gamma$ & $67,5^{\circ}-\frac{3}{8} \gamma$ & $\gamma$ & $\gamma<36^{\circ}$ \\
\hline 4 & $3 / \mathrm{a}$ & $150^{\circ}-\frac{7}{6} \gamma$ & $30^{\circ}+\frac{1}{6} \gamma$ & $\gamma$ & $\gamma<36^{\circ}$ \\
\hline 5 & $3 / \mathrm{b}$ & $360^{\circ}-7 \gamma$ & $6 \gamma-180^{\circ}$ & $\gamma$ & $36^{\circ}<\gamma<\frac{270^{\circ}}{7}$ \\
\hline 6 & 4 & $120^{\circ}-\frac{2}{3} \gamma$ & $60^{\circ}-\frac{1}{3} \gamma$ & $\gamma$ & $\gamma<45^{\circ}$ \\
\hline 7 & $5 / \mathrm{a}$ & $157,5^{\circ}-\frac{7}{8} \gamma$ & $22,5^{\circ}-\frac{1}{8} \gamma$ & $\gamma$ & $\gamma<20^{\circ}$ \\
\hline 8 & $5 / \mathrm{b}$ & $7 \gamma$ & $180^{\circ}-8 \gamma$ & $\gamma$ & $\frac{90^{\circ}}{7}<\gamma<20^{\circ}$ \\
\hline 9 & $6 / \mathrm{a}$ & $120^{\circ}-\frac{1}{3} \gamma$ & $60^{\circ}-\frac{2}{3} \gamma$ & $\gamma$ & $\gamma<36^{\circ}$ \\
\hline 10 & $6 / \mathrm{b}$ & $90^{\circ}+\frac{1}{2} \gamma$ & $90^{\circ}-\frac{3}{2} \gamma$ & $\gamma$ & $\gamma<36^{\circ}$ \\
\hline 11 & 7 & $180^{\circ}-4 \gamma$ & $3 \gamma$ & $\gamma$ & $\gamma<22,5^{\circ}$ \\
\hline 12 & 8 & $180^{\circ}-\frac{7}{3} \gamma$ & $\frac{4}{3} \gamma$ & $\gamma$ & $\gamma<\frac{270^{\circ}}{7}$ \\
\hline 13 & 10 & $180^{\circ}-\frac{5}{2} \gamma$ & $\frac{3}{2} \gamma$ & $\gamma$ & $\gamma<36^{\circ}$ \\
\hline 14 & 11 & $180^{\circ}-5 \gamma$ & $4 \gamma$ & $\gamma$ & $\gamma<18^{\circ}$ \\
\hline 15 & 12 & $135^{\circ}-\frac{7}{4} \gamma$ & $45^{\circ}+\frac{3}{4} \gamma$ & $\gamma$ & $\gamma<\frac{180^{\circ}}{7}$ \\
\hline 16 & 20 & $180^{\circ}-7 \gamma$ & $6 \gamma$ & $\gamma$ & $\gamma<\frac{90}{7}$ \\
\hline 17 & $21 / \mathrm{a}$ & $135^{\circ}-\frac{1}{2} \gamma$ & $45^{\circ}-\frac{1}{2} \gamma$ & $\gamma$ & $\gamma<30^{\circ}$ \\
\hline 18 & $21 / \mathrm{b}$ & $90^{\circ}+\gamma$ & $90^{\circ}-2 \gamma$ & $\gamma$ & $\gamma<30^{\circ}$ \\
\hline 19 & 24 & $135^{\circ}-\gamma$ & $45^{\circ}$ & $\gamma$ & $\gamma<45^{\circ}$ \\
\hline 20 & 26 & $6 \gamma$ & $180^{\circ}-7 \gamma$ & $\gamma$ & $15^{\circ}<\gamma<22,5^{\circ}$ \\
\hline 21 & 27 & $180^{\circ}-6 \gamma$ & $5 \gamma$ & $\gamma$ & $\gamma<15^{\circ}$ \\
\hline 22 & 28 & $135^{\circ}-\frac{3}{2} \gamma$ & $45^{\circ}+\frac{1}{2} \gamma$ & $\gamma$ & $\gamma<30^{\circ}$ \\
\hline 23 & 29 & $180^{\circ}-3 \gamma$ & $2 \gamma$ & $\gamma$ & $\gamma<30^{\circ}$ \\
\hline
\end{tabular}




\section{Answer for the original problem}

Finally, we give a spectacular geometric answer for the generic question of our article (Figure 20). On the picture, with $\alpha$ on the horizontal axis and $\beta$ on the vertical axis $(\alpha \geq \beta \geq \gamma)$, a grey triangle shows every possible acute triangles and the right triangles can be seen on the left side of this grey triangle. They can all be formed as a combination of 3 isosceles triangles. The obtuse triangles that can be formed the same way are on a line segment in the middle (some may be on more than one).

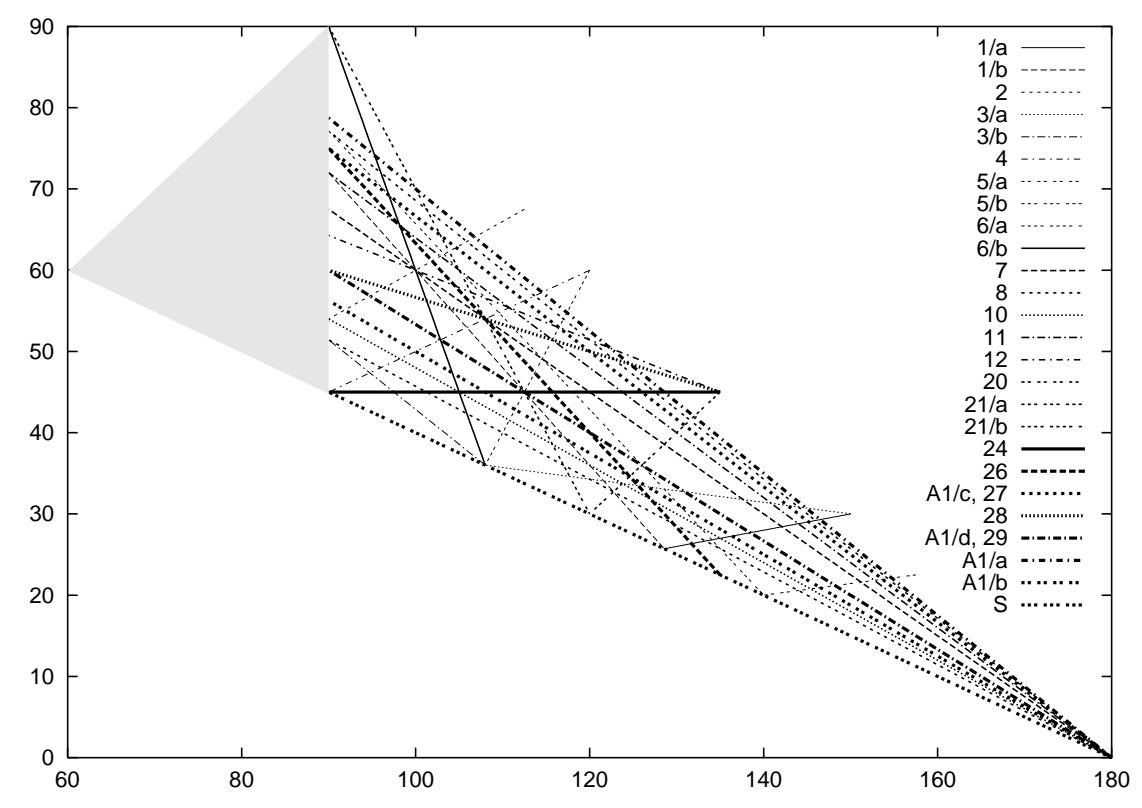

Figure 20.

The "short cut" cases 27 and 29 of the above 23 different types generate two such triangle types which we could already form without dividing the obtuse angle (cases A1/c and A1/d). Cases A1/a and A1/b, on the other hand, are completely different from all computer-generated case; they are also on our summarizing figure. Finally, the trivial symmetric case is denoted by S. 


\subsection{Counterexamples}

It is also clear that our result gives a necessary and sufficient condition for the existence of a decomposition. That is, after reviewing all possible cases it is easy to create triangles that cannot be decomposed into three isosceles triangles (as it can be seen, an infinite number of essentially different such triangles can be found).

In all these cases $\gamma<45^{\circ}$. In most cases (except for two) $\gamma<40^{\circ}$ also holds: the equality $\gamma=40^{\circ}$ is allowed only in Solution 4 and Solution 24 . Let us assume this. Based on Solution $4, \beta=60^{\circ}-\frac{\gamma}{3}=46, \dot{6}$ can be excluded. The same can be done with $\beta=45^{\circ}$ based on Solution 24 . Now any angle $\beta$ will be "adequate" for which $40^{\circ}<\beta<50^{\circ}, \beta \neq 45^{\circ}$ and $\beta \neq 46, \dot{6}$. Now if, for example, $\beta=41^{\circ}$, then $\alpha=99^{\circ}$, that is an obtuse triangle with angles $\alpha=99^{\circ}, \beta=41^{\circ}, \gamma=40^{\circ}$ cannot be decomposed into three isosceles triangles.

Another construction: It has been shown that $\alpha$ and $\beta$ are some rational multiple of $\gamma$. This follows from having only rational coefficients in our equations and inequalities. It is clear that there are infinitely many obtuse triangles for which the ratio of their smallest and two larger angles is irrational. One such construction is $\gamma=30^{\circ}, \beta=x \cdot \gamma, \alpha=(5-x) \cdot \gamma$, where $x$ is an arbitrary irrational number between 1 and 2 , for example $\sqrt{2}$. Thus, say, a triangle with angles $\alpha \approx 107,5737^{\circ}, \beta \approx 42,4263^{\circ}, \gamma=30^{\circ}$ cannot be decomposed into three isosceles triangles, either.

\section{The graphical representation of computer-generated solutions}

\subsection{Utilities}

Three more utilities have been used.

- The representation of the vast number of computer-generated solutions is best done with a computer, too. The results of the analytic geometrical calculations below are finally converted into a postscript file, which can be printed virtually with any printer and watched on any monitor regardless of its resolution. The main advantage of postscript language is that the viewers are also free to access.

- It is useful to graph the solutions in an $(\alpha, \beta)$ coordinate system. For simplicity, the easy-to-use gnuplot programme is employed, which is suitable for creating automatically generated graphs. 
- The graphs can also be interpreted by typesetting programmes: this is important for the final form of the publication. We used ${ }^{A} T_{E} X$ which is a freeware.

All our previous apparatus is utilized here: the system of equations we get is solved again by Maxima; the program parts that create the postscript and gnuplot codes are also written in Pascal; and the communication between the two is provided by the Linux operating system.

\subsection{Analytical geometric calculations}

Let the points $(a, b)$ and $(c, d)$ and the acute angles $\alpha$ and $\beta$ be given on the plane. Measure angles $\alpha$ and $\beta$ in the endpoints of the line segment connecting the two points, and denote the coordinates of the intersection of the resulting two half-lines with $(e, f)$ (Figure 21). Our task was to produce an algorithm which calculates $e$ and $f$ from the original data.

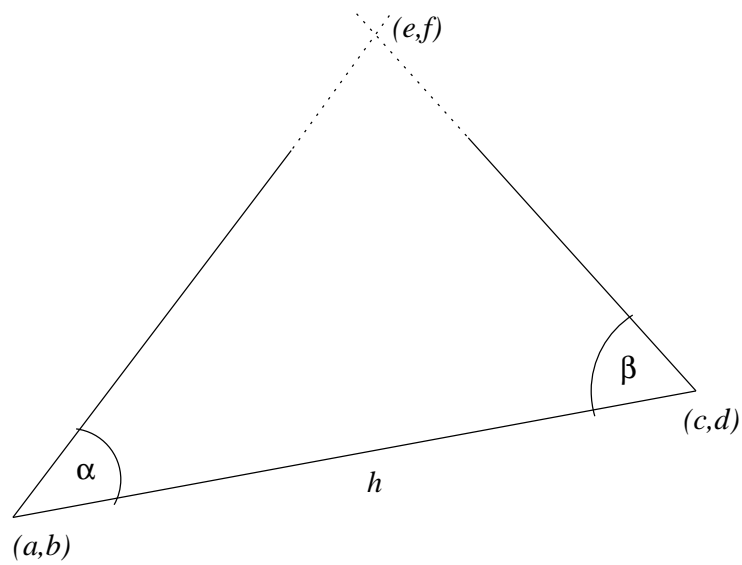

Figure 21.

First, the equation of the given line segment is determined. Let $n=d-b$, $m=a-c$; now it is clear that for the length $h$ of this line segment $h=\sqrt{n^{2}+m^{2}}$. For the direction vector $\left(n^{\prime} m^{\prime}\right)^{T}$ of the line segment: $n^{\prime}=\frac{n}{h}$. The line containing the line segment will be the set of exactly those points $(x, y)$ for which $n^{\prime} x+m^{\prime} y=$ $n^{\prime} a+m^{\prime} b$, that is,

$$
n^{\prime}(x-a)=m^{\prime}(b-y)
$$


Denote the direction vectors of the half-lines starting from point $(a, b)$ and $(c, d)$ with $\left(n^{\prime \prime} m^{\prime \prime}\right)^{T}$ and $\left(n^{\prime \prime \prime} m^{\prime \prime \prime}\right)^{T}$, respectively. It is obvious that

$$
\left(\begin{array}{c}
n^{\prime \prime} \\
m^{\prime \prime}
\end{array}\right)=\left(\begin{array}{c}
n^{\prime} \\
m^{\prime}
\end{array}\right) \cdot\left(\begin{array}{cc}
\cos \alpha & -\sin \alpha \\
\sin \alpha & \cos \alpha
\end{array}\right)
$$

and

$$
\left(\begin{array}{c}
n^{\prime \prime \prime} \\
m^{\prime \prime \prime}
\end{array}\right)=\left(\begin{array}{c}
n^{\prime} \\
m^{\prime}
\end{array}\right) \cdot\left(\begin{array}{cc}
\cos (-\beta) & -\sin (-\beta) \\
\sin (-\beta) & \cos (-\beta)
\end{array}\right)
$$

as these vector are the result of the rotation of vector $\left(n^{\prime} m^{\prime}\right)^{T}$. Therefore the equations of these half-lines are:

$$
\begin{aligned}
n^{\prime \prime}(x-a) & =m^{\prime \prime}(b-y), \\
n^{\prime \prime \prime}(x-c) & =m^{\prime \prime \prime}(d-y) .
\end{aligned}
$$

Interpreting these two equations as a system of equations for $x$ and $y$ we get the coordinates $e=x, f=y$ as its solution.

\subsection{The actual graphing of the solutions}

Triangle $A B C$ can be easily graphed with the help of a computer now. Take points $B$ and $C$ arbitrarily (here these will be points $(0,0)$ and $(1,0)$ ), connect them with a line segment, then measure angles $a$ and $c$ in the two endpoints. The intersection of the two legs of the angle is point $A$. The coordinates can be calculated as seen in Section 5.2. Line segments $B A$ and $C A$ can be drawn now.

In the following we deal with the "long cut" case. (The "short cut" case can be done similarly.) The coordinates of point $D$ has to be determined. Therefore we measure angles $c$ and $e$ on line segment $A C$ at points $C$ and $A$, respectively. The intersection of the two legs of the angle (also determined as seen in Section 5.2) gives the coordinates of $D$ and so line segment $A D$ can also be drawn.

One of the endpoints of the line segment dividing triangle $A C D$ is $E$, but the other endpoint depends upon which angle was divided. Table 2 (which is used in the computer programme, too) helps to decide in each case which angles should be measured at the endpoints of which line segment, and which line segments decompose triangle $A C D$. 


\begin{tabular}{|c|c|c|c|}
\hline Decomposed angle & Starting line segment & Angles to be measured & $\begin{array}{c}\text { Line segment } \\
\text { to be drawn }\end{array}$ \\
\hline \hline$c$ & $C A$ & $i, e$ & $C E$ \\
\hline$e$ & $C A$ & $c, h$ & $A E$ \\
\hline$g$ & $A D$ & $e, h$ & $D E$ \\
\hline
\end{tabular}

Table 2:

\section{Technical details of the computer processing}

\subsection{Maxima}

Version 5.9 works on the current Linux systems without any trouble. The programme can be downloaded from [3].

With the help of Maxima we solve the system of equation at 3.2 with the following command line:

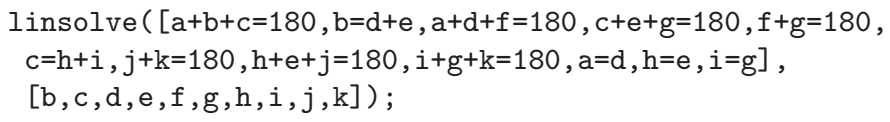

Thus, each unknown variable is expressed with $a$. If we need the result in decimal form, the command line

float (\textrm\{expression\});

is used. The output received from the

$$
\text { display2d(false) }
$$

command line is transferred to the calling programme without line breaks, while the output must be forwarded to the calling process this way. In addition,

$$
\text { linel (2000); }
$$

command ensures that the output fits into one line.

\subsection{Pascal}

Version 1.0.10 can be downloaded from [4].

Each computational task is stored in a character string. 


\subsection{Postscript}

We receive a code similar to the postscript extract below as the output of the Pascal programme:

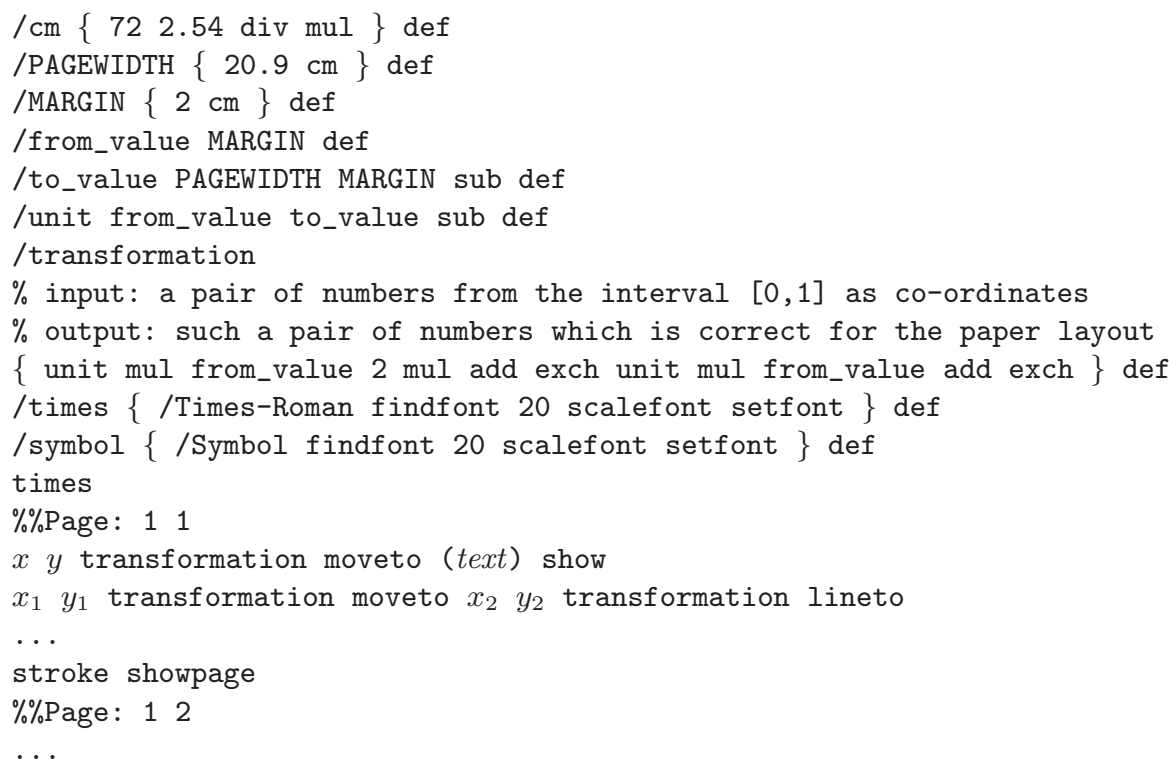

Starting from the point with coordinates $(x ; y)$ this part of the programme prints the text text on the screen and draws the line segment between points $\left(x_{1} ; y_{1}\right)$ and $\left(x_{2} ; y_{2}\right)$. We define two fonts: with Symbol we produce the Greek letter $\gamma$. Each decomposition is on a different page, they are organized later by a programme called pstops so that more than one picture fit on one page.

pstops ([6]) and ghostview ([5]) are usually included in every Linux distribution (the latter being the most popular postscript viewer). The postscript output can be processed by $\mathrm{LT}_{\mathrm{EX}}$ without any trouble: this is how this publication was made.

\subsection{Gnuplot}

Two solutions can be compared with the following code (we used Version 3.7; its description can be found at [8]).

set term postscript

set parametric

a1 $=0.000$ 


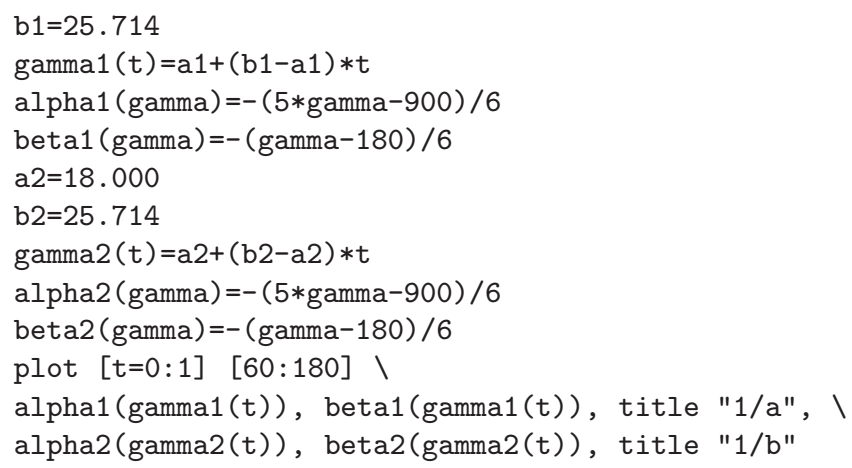

In the first line we determine that we expect postscript output. The second line sets the parametric drawing mode. The numbers $a N$ and $b N$ give the intervals prescribed for $\gamma$. The actual drawing is done by the plot command with representation in interval $\alpha \in[60,180]$ with captions " $1 / \mathrm{a}$ " and " $1 / \mathrm{b}$ ". The output of the programme was similarly produced with Pascal.

\subsection{Downloadable material}

The programme completely solving the problem can be downloaded from [7] with programme description in the README file.

\section{References}

[1] J. Kosztolányi Z. Kovács and E. Nagy, Decomposition of Triangles into Isosceles Triangles I, Teaching Mathematics and Computer Science 2 (1) (2004), 163-184.

[2] A. Soifer, How Does One Cut Triangle?, Center for Excellence in Mathematical Education, Colorado Springs, 1990.

[3] http://maxima.sf.net.

[4] http://www.freepascal.org.

[5] http://www.cs.wisc.edu/ ${ }^{\sim}$ ghost.

[6] http://www.dcs.ed.ac.uk/home/ajcd/psutils/pstops.html.

[7] http://www.math.u-szeged.hu/ ${ }^{\sim}$ kovzol/Articles/Decomposition/II.

[8] http://www.ucc.ie/gnuplot/gnuplot-faq.html.

JÓZSEF KOSZTOLÁNYI

UNIVERSITY OF SZEGED

BOLYAI INSTITUTE

H-6701 SZEGED, ARADI VÉRTANÚK TERE 1

HUNGARY 


$$
\text { "kosztolanyi" — 2005/2/17 — 17:02 — page 300 — \#26 }
$$

300 Kosztolányi, Z. Kovács and E. Nagy : Decomposition of triangles into isosceles triangles II

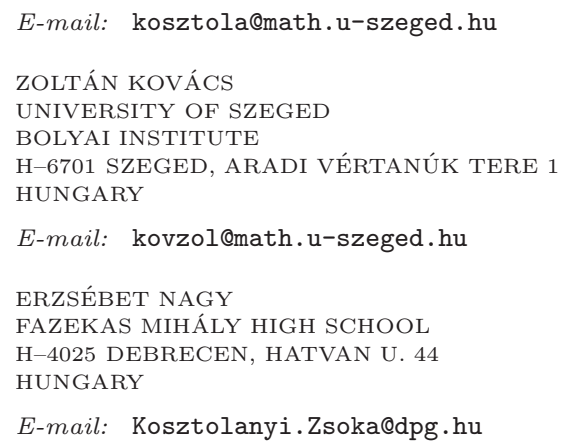

(Received March, 2004) 